\title{
Development and Maintenance of a Cross-mixed Mating System in the Orchid Bulbophyllum orientale
}

\section{Li-Jun Chen ${ }^{1}$, Guo-Qiang Zhang ${ }^{1}$, Li-Qiang Li', Yu-Ting Zhang ${ }^{1}$, Wen-Hui Rao' ${ }^{1}$ and Zhong-Jian Liu'-3*}

${ }^{1}$ Shenzhen Key Laboratory for Orchid Conservation and Utilization, The National Orchid Conservation Center of China and The Orchid Conservation \& Research Center of Shenzhen, Shenzhen 518114, China

${ }^{2}$ The Center for Biotechnology and BioMedicine, Graduate School at Shenzhen, Tsinghua University, Shenzhen 518055, China

${ }^{3}$ College of Forestry, South China Agricultural University, Guangzhou 510642, China

\begin{abstract}
Outbreeding is usually advantageous because inbreeding suffers from depression. Nevertheless, mixed mating is very common in nature. We found two co-existing plant types, self-compatible and self-incompatible, in populations of the orchid Bulbophyllum orientale. The floral parts of this plant form a device to promote cross-pollination. Rancid substances are excreted to lure pollinators to the labellum, and pollens are attached to pollinators through a delicate mechanism. Given that many inflorescences and flowers are present on a clone and each inflorescence, respectively, pollinating insects may continuously visit inflorescences of the same clone and flowers of the same inflorescence but rarely continuously visit different populations separated by large distances. Consequently, self-compatible plants produce seeds from both crossing and selfing, and self-incompatible plants only bear crossing seeds. Thus, a crossmixed mating system is created in the population. Individuals capable of producing both crossed and selfed seeds have better chances in natural selection. The strict crossing system is broken down, and a cross-mixed mating system consisting of both mixed mating and strict crossing is formed. The cross-mixed mating system fluctuates with varying behavior of pollinating insects. The mixed mating system is favored because a population has many clone individuals and because each individual has many multi-flower inflorescences in $B$. orientale. The partial strict crossing is retained, and it can counteract the latent harm caused by selfing and assist in the maintenance of this cross-mixed mating system. The successful evolution of flowering plants is demonstrated by the mode of attraction for pollinating insects, the smart use of the cross-pollinating facility, and the tradeoff between crossing and mixed mating.
\end{abstract}

Keywords: Bulbophyllum orientale; Pollinating behavior; Crossmixed mating system; Tradeoff; Self-incompatibility; Self-evolution

\section{Introduction}

Plant breeding systems comprise inbreeding, outbreeding, and apomixes [1]. Among these systems, outbreeding is considered the primary driver of pollination diversity. Flowering plants have developed many mechanisms, such as dioecy, dichogamy, herkogamy, self-incompatibility [2], and flexistyly [3,4], to avoid inbreeding and promote outbreeding. Selfing has many benefits, such as opening up new habitats $[5,6]$, avoiding unresponsive pollinators [7-10], and automatically transferring genes to offspring [11]. However, these benefits are severely abated because of the high mating costs incurred from the pollen and ovule discounting. Angiosperm evolution has recurrent alternating dominance of cross-pollination and selfpollination [12]. The inbreeding depression caused by self-pollination is deemed to be a selective pressure in the evolution of plant breeding systems [4,7]. Nevertheless, the reproduction assurance of selfing has a greater effect than the inbreeding depression in facilitating the evolution of mating systems. Consequently, the plant may adopt a mixed mating system as a tradeoff between crossing and selfing [13]. Geitonogamy is related to the evolution of inflorescence [14] and pollinator behavior [13]. These relationships can influence population dynamics $[15,16]$. Some plants maintain their mixed mating systems with strict crosspollination in single flowers and geitonogamy in the inflorescence [17]. The mixed mating system is a compromise between the plant and the environmental conditions [18]. Inbreeding depression is a selective pressure that thwarts the fixation of selfing-favorable genotypes, prevents self-evolution [19], and therefore preserves the mixed mating system in the long term [20].

The great diversity of pollination mechanisms in angiosperms is most evident in breeding systems of animal pollination [21]. The changes in pollination environment and pollinator behavior directly affect plant mating $[6,9,13]$. Natural selection is believed to render the floral structure adaptive to cross-fertilization [22,23]. Orchidaceae, an advanced evolutionary family of angiosperm, has a highly specialized floral structure for insect pollination. Although orchids have developed many techniques for cross-pollination [3,6,15,23-27], many species use mixed mating as a breeding strategy [28]. Recently, Bulbophyllum orientale was found to have many large clone individuals, each with many multi-flower inflorescences. However, 3.5\% of individuals within a single population have a very low natural fruit ratio, and preliminary selfing tests show that they are all self-incompatible. The type of breeding system used by $B$. orientale is a subject worth extensive research. Given that pollination pattern and pollinator feeding behavior affect development of the breeding system, this study aims to examine the pollination mechanism, including flowering phenology, flower structure, stigma receptivity, pollen activity and floral odor and breeding system of Bulbophyllum orientale, to investigate the evolutionary dynamics of the mating system of $B$. orientale, and to reveal the development and maintenance of its breeding system diversity.

\section{Methods}

\section{Studied plant and observation site}

B. orientale is an Asian Orchidaceae species that is distributed

*Corresponding author: Zhong-Jian Liu, Shenzhen Key Laboratory for Orchid Conservation and Utilization, The National Orchid Conservation Center of China and The Orchid Conservation \& Research Center of Shenzhen, Shenzhen 518114, China, Tel.: 86-755-25712359; Fax: 86-755-25711928; E-mail: liuzj@sinicaorchid.org

Received March 30, 2014; Accepted March 31, 2014; Published April 10, 2013

Citation: Chen LJ, Zhang GQ, Li LQ, Zhang YT, Rao WH (2014) Development and Maintenance of a Cross-mixed Mating System in the Orchid Bulbophyllum orientale. J Phylogen Evolution Biol 2: 124. doi:10.4172/2329-9002.1000124

Copyright: ( 2014 Chen LJ, et al. This is an open-access article distributed under the terms of the Creative Commons Attribution License, which permits unrestricted use, distribution, and reproduction in any medium, provided the original author and source are credited. 
in southern Yunnan, China, and Thailand, and grows on the trunks of broadleaf forests [29]. It is a perennial plant with adnate racemes. Approximately 10 to 70 flowers grow compactly and open on the same day in each inflorescence. The flowers are bisexual and can last for $5 \mathrm{~d}$ to $9 \mathrm{~d}$ if unfertilized. The florescence of this plant is from June to October. Observations and experiments were carried out both in natural populations of $B$. oriental at the evergreen broadleaf forest in Pu'er, Yunnan, China $\left(22^{\circ} 33^{\prime} \mathrm{N}, 101^{\circ} 17^{\prime} \mathrm{E}, 1200 \mathrm{~m}\right.$ a.s.l. $)$ and in the artificial populations in The National Orchid Conservation Center of China at Shenzhen, China $\left(22^{\circ} 35^{\prime} \mathrm{N}, 114^{\circ} 10^{\prime} \mathrm{E}, 50 \mathrm{~m}\right.$ a.s.l. $)$.

\section{Observation of flowering phenology and floral structural features}

Observation of flowering phenology: The flowering phenology of all plants in natural populations was observed from 2004 to 2008. Different flowering stages, namely, early, full, and late, were recorded. Each year, 20 unopened inflorescences were randomly marked and observed. For each single flower, the opening time, the corolla withering time, and the shape changes of the labellum and corolla were recorded. Labellum and stigma shapes and positional relationship between the anther and stigma

Freshly opened flowers were collected, and perianths were partly removed. The labellum and stigma shapes, as well as the positional relationship between the anther and stigma, were examined and photographed under a stereomicroscope.

\section{Flowering biology characteristics}

Histochemistry of the labellum secretion: Ten flower labella from different inflorescences were randomly collected, immersed in the staining solution for $10 \mathrm{~min}$, and observed under a microscope. A blue or black color on the labellum surface after staining with iodine potassium iodide (IKI) indicates the presence of starch, and a red color after staining with Sudan IV confirms lipid secretion [30]. Each experiment was repeated five times, and the colors were recorded.

Stigma receptivity, pollen activity, and seed-setting rate at different pollination periods: Fresh flowers were randomly collected at different growth stages (e.g., at $0,0.5,1,2,3 \ldots 10 \mathrm{~d}$ after opening). Pollen activity and stigma receptivity were tested by the 3-(4,5-dimethylthiazol-2-yl)-2,5-diphenyltetrazolium (MTT) method [31]. Half of the fresh pollinium and half of the column (stigma, longitudinal cutting) were placed on the slide. The other halves of both samples were placed in water in a vessel, boiled for $15 \mathrm{~min}$, and then placed on the slide for comparison. One drop of MTT solution was added to the sampled pollinium and stigma. After the samples were thoroughly mixed and air-dried, another drop of MTT solution was added. The coloration of the pollinium and stigma was observed under a microscope after the samples were completely dried. A blue or black color of the pollinium indicates positive pollinium activity and stigma receptivity. A yellowish brown color or no color change indicates pollen inactivity and no stigma receptivity.

The following experiments were performed to determine the fruit-set rate of $B$. orientale at different pollination periods. In the first experiment, the flowers were bagged before opening. These flowers were artificially cross-pollinated at $1,2,3,4,5,6,7$, and $8 \mathrm{~d}$ after opening using pollen grains from flowers of different plants that had opened for 1 or $2 \mathrm{~d}$ and then bagged again. In the second experiment, the flowers were bagged before opening. They were artificially crosspollinated at $1,2,3,4,5,6,7$, and $8 \mathrm{~d}$ after opening (or when the corolla withered) with pollen grains from different flowers on the same inflorescence (geitonogamy) and then bagged again. The fruit-set rate in each experiment was calculated.

Floral odor: Five plants bearing B. orientale flowers were randomly selected from the populations. The inflorescences were separately placed in $100-\mathrm{mL}$ bottles, which were then sealed. The odor was extracted with CAR/PDMS $75 \mu \mathrm{m}(30 \mathrm{~min})$, desorbed $\left(3 \mathrm{~min}, 200^{\circ} \mathrm{C}\right)$, and then analyzed on a Finnigan TRACE GC-MS $\left(25^{\circ} \mathrm{C}, 65 \%\right.$ humidity). The volatile components were analyzed at The Analysis Center of South China Agricultural University.

\section{Visiting insects and their visiting behavior}

Species and visiting behavior of the visiting insects: Each year, 10 opened inflorescences were randomly selected from the populations, marked, and continuously observed for $2 \mathrm{~d}$ from 8:00 to 19:00. The number of visiting insects on each marked inflorescence was counted, and the visiting behavior of each insect species was described, photographed, and videoed. The following items were recorded for each inflorescence: the type of visiting insect, the visit frequency, the visit duration, and the number of flowers visited by one visitor at one time. Specimens of the insects were also captured.

Attraction of floral features to pollinating insects: Ten freshly opened self-compatible inflorescences in the populations were marked to examine the attraction of floral features to pollinating insects. The labella were removed in five inflorescences, and the visiting frequency and visiting behavior of the pollinating insects to all inflorescences were compared. This experiment was performed three times.

\section{Breeding system}

Artificial cross-pollination experiments of self-compatible and self-incompatible plants were performed during the flowering seasons of $B$. orientale from 2004 to 2008 . Each of the following experiments was performed every year on a sample set of $6 \times 15$ flowers: $(1)$ no treatment, labeled until the plants fructified; (2) soon before opening, the pollen grains were removed, and the flowers were bagged; (3) the flowers were bagged before opening, artificially cross-pollinated (with different plants) after opening, and then bagged again; (4) the flowers were bagged before opening, artificially self-pollinated (geitonogamy) after opening, and then bagged again; and (5) the flowers were bagged before opening and then stored until they withered. The numbers of flowers and inflorescences were recorded. The fruit-set rate of each group was calculated.

\section{Inbreeding depression}

From 2004 to 2008, eight fruits at maturity stage were randomly selected each year from the cross- and self-pollination experiments of the inbreeding system examinations (a total of 40 fruits over 5 years for each experiment), and the seed number in each fruit was counted. The seeds of a cross-fruit and a self-fruit were paired into a sample group and separately sown on the same artificial culture medium. After germination, both the budded and un-budded seeds were counted. The seed number and the average seed germination rate from the self- and cross-offspring were examined in pairs by T-test or $\mu$-test to calculate the benefit and the degree of inbreeding depression [32-34].

\section{Cross-pollinator shortage rate and selfing rate}

Given that many inflorescences and flowers are present on a clone and each inflorescence, respectively, we need to examine the degree of geitonogamy to compare their fruit ratios, inferring pollinator's shortage rate. We hypothesize that all of the flowers in a many-flowered inflorescence should be pollinated when pollinators are sufficient, generating the highest fruit ratio. We used the artificial pollination to 
imitate the condition of enough pollinators and determine the highest possible fruit ratio. Theoretically, all of the flowers should fruit after pollination, giving a fruit ratio of $100 \%$. The pollinator storage rate is derived by subtracting the fruit ratio in the natural condition from the theoretical fruit ratio and then dividing by the highest possible fruit ratio. In the same way, the pollinator storage rate within a population and among populations can be calculated by comparing artificial pollination and the natural fruit ratio.

The following examinations of the cross-pollinator shortage rate and selfing rate were carried out from 2006 to 2008.

Within inflorescences: Each year, among the 10 populations, 10 self-incompatible inflorescences (or self-compatible inflorescences whose stamens were removed) were paired with 10 self-compatible inflorescences. These inflorescences were pollinated by pollinators under natural conditions. Another 10 inflorescences were artificially cross-pollinated. The following attributes were calculated: pollinator shortage rate of inflorescences $=1-$ natural fruit-set rate of selfcompatible flowers / fruit-set rate of artificial cross-pollination; inflorescence selfing-rate $=$ natural fruit-set rate - natural fruit-set rate of self-incompatible inflorescences (self-compatible inflorescences with stamens removed); inflorescence crossing-rate $=$ natural fruit-set rate of self-incompatible inflorescences (self-compatible inflorescences with stamens removed); and cross-pollinator shortage rate of inflorescences $=1-$ natural fruit-set rate of self-incompatible inflorescences (selfcompatible inflorescences with stamens removed).

Within population: In the 10 populations, 10 inflorescences each year were bagged before flower opening, had their stamens removed after flower opening (two inflorescences from each population), and were paired with whole flowers from another 10 inflorescences (two inflorescences from each population). All flowers were then pollinated under natural conditions. The following attributes were calculated: pollinator shortage rate within population $=1-$ natural fruit-set rate of whole flowers / artificial crossing fruit-set rate of whole flowers; and selfing (inbreeding) rate within population (mating between flowers in the same population) $=$ natural fruit-set rate of whole flowers - fruit-set rate of stamenless flowers among populations (the results from Section "Among populations" were used).

Among populations: $B$. orientale is pollinated by very small insects. Can these pollinators pollinate among populations and be used to test effect of outbreeding of $B$. orientale? In two adjacent populations located $30 \mathrm{~m}$ apart from each other, all inflorescences of one population were bagged each year before flower opening, had their stamens removed after flower opening, and were then paired with whole inflorescences from the other population to receive natural pollination. The following attributes were calculated: pollinator shortage rate among populations $=1-$ fruit-set rate of stamenless flowers; and crossing (outbreeding) rate among populations $=$ fruit-set rate of stamenless flowers .

\section{Results}

\section{Shape and structure of flowers and flowering phenology characteristics}

Shape, structure and flowering phenology: Each clonal series of B. orientale can include up to 300 to 400 related individuals, and each individual can simultaneously produce two to three inflorescences. The flowering season begins in early June. Full blossom is in mid-July, and late blossom is in early October. The flower opens when the scape has grown to $10 \mathrm{~cm}$ to $20 \mathrm{~cm}$ in length. Under natural conditions, an unpollinated single flower has a blossom that lasts for $7.3 \pm 2.3 \mathrm{~d}$ $(n=50)$, and an unfertilized flower will completely fall off from the rachis. The ovary begins to swell $2 \mathrm{~d}$ after pollination. After a 2 month gestation, the capsule matures and splits to scatter the seeds.

Labellum and stigma shapes and positional relationship between the anther and stigma: The labellum of $\mathrm{B}$. orientale is attached to the column base with a movable joint, to form a see-saw lip of $4 \mathrm{~mm}$ in length and $2 \mathrm{~mm}$ in width. The fore lip has a groove in the middle that connects to the shallow spoon-shaped concavity on the back lip. The mastoid glands on the groove fringe secrete mucous substances that flow into the concavity on the back lip. The stigma is largely cylindrical, ca. $3 \mathrm{~mm}$ long and $2 \mathrm{~mm}$ wide, and has one $1.5 \mathrm{~mm}$ long column tooth on both sides at the top. The stigma cavity has an ovate-elliptic shape, and it is located at the upper part of the column. It is $1.4 \mathrm{~mm}$ to $1.5 \mathrm{~mm}$ long and $0.8 \mathrm{~mm}$ to $0.9 \mathrm{~mm}$ wide, and it is usually full of a glutinous substance after flower opening Figure 1A. Four elliptic pollinaria (two pairs, each with no viscid disc) are exposed when the anther cap on the lateral fringe of stigma cavity splits off during flower opening Figures $1 \mathrm{~B}$ and $1 \mathrm{C}$. A lip-shaped rostellum exists between the stigma cavity and the anther bed. The lower surface of the rostellum breaks upon gentle squeezing and exudes glutinous substances Figure 1D.

\section{Flowering biology characteristics}

Histological chemistry of secretory glands on labellum: After staining with IKI, the original color of the labellum persisted, and
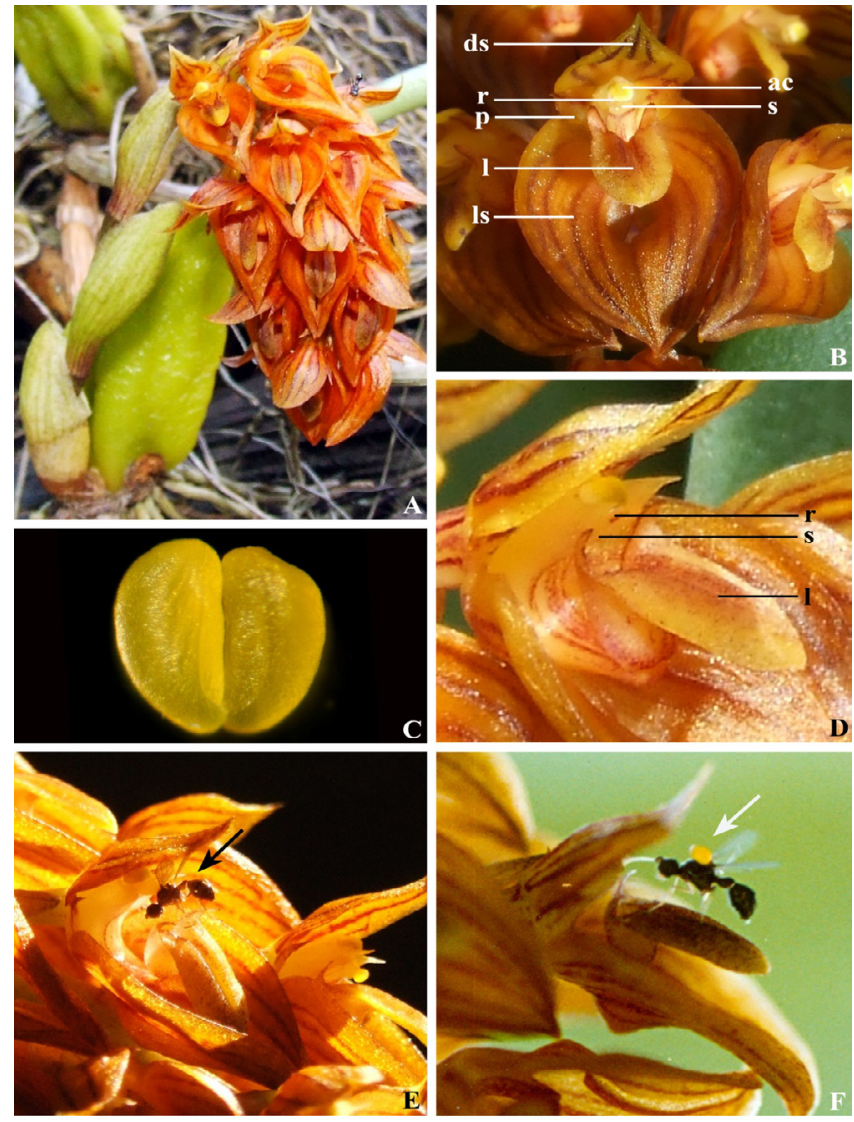

Figure 1: Floral morphology of Bulbophyllum orientale and its pollination by Sepsidae species. (A) Inflorescence. (B) Front view of the flower: ac, anther cap; r, rostellum; I, labellum; ds, dorsal sepal; Is, lateral sepal; p, petal; and s, stigma. (C) Pollinarium. (D) Side view of the flower showing the relative position of labellum, rostellum, and stigma: $r$, rostellum; I, labellum; and s, stigma. (E) Sepsidae species entering into a flower (arrow). (F) Sepsidae species exiting a flower, carrying pollinaria on its thorax (arrow). 
neither blue nor black color appeared. After Sudan IV staining, the papillate glands at the groove fringe of the labellum developed a red color. The red color stretched along the groove to the lip concavity. This result indicates the presence of secretory glands on the labellum and the secretion of lipid substances.

Fruit-set rate, pollen activity, and stigma receptivity at different pollination periods: All sampled pairs of pollinium and stigma were stained blue-black, and the reference pairs showed no color change. Therefore, the pollen was active, and the stigmas were receptive during the flowering season. No dichogamy was observed.

The fruit-set rates at different pollination periods are summarized in Table 1. Before flower fading, the cross-pollination of all flowers had high affinity, and the self-pollination exhibited both self-compatibility and self-incompatibility among plants, regardless of the pollination period.

Odor analysis: According to the gas chromatogram of the volatile elements from $B$. orientale Figure 2 and the NIST database, the odor of $B$. orientale contained sesquiterpene, $a$-copaene, and several lipoids. These substances are the food of pollinating insects and produce rancid smells [35].

\section{Visiting insects and their visiting behavior}

Visiting insects and their visiting mode: A $2 \mathrm{~d}$ (from 8:00 to 19:00) point observation was carried out each year between June and October of 2004 to 2008 (a total of $120 \mathrm{~h}$ observation). Five plants (each ca. 200 flowers) were observed during each observation period; thus, 25 plants (ca. 5000 flowers) were observed in 5 years. Successful visits of Sepsidae species, Phytomyza horticola, and Drosophilidae species to the flowers were observed. The average visit frequency was 2.0 times per $60 \mathrm{~min}$ for each inflorescence. When the $B$. orientale flower opened, the glands on the groove fringe of the labellum excreted glutinous substances with strong smells of putrid berry to allure Sepsidae species, $P$. horticola, and Drosophilidae species. The visitor landed on the upper part of the labellum and pushed the labellum downwards to make an interstice between the lower labellum and the column. The visitor then foraged into the interstice and became clamped on its head and chest as the labellum closed upwards because of the reduced pressing weight. The ridged back of the visitor became pressed into the stigma cavity Figure 1E. When the visitor struggled to escape, it had to exit through the entry route because of the obstacles of the labellum lobelet and column arm. It stretched its belly upwards and kicked the upper labellum with its back legs to increase the space between the labellum and the column. The limited flexibility of the labellum forced the visitor to break the rostellum with its chest and back and carry the glutinous secretion with the uncovered pollinium before it could escape Figure 1F. When the pollinium-carrying insect repeated the above process on another flower, the foreign pollinium on the insect's back was smeared into the stigma cavity when the insect attempted to exit. Then, the new pollinium was passed on and taken away, thereby achieving cross-pollination. Given that the $B$. orientale raceme has many flowers, some visitors foraged on other plants and effectuated cross-pollination after having escaped from one flower. Nevertheless, many more visitors continued to forage on the same inflorescence or the same clonal plant, resulting in the crosspollination of the same plant.

Attraction of the labellum to the pollinating insects: In the $60 \mathrm{~h}$ observations, the flowers whose labella were removed had a pollinator visiting frequency of zero, whereas the pollinator visiting frequency of natural flowers (all visitors included) was, on average, 2.0 times per $60 \mathrm{~min}$ for each inflorescence. After the flowering season ended, the fruit-set rate of the flowers without labellum was zero, whereas that of the natural flowers was $10.89 \% \pm 6.43 \%(n=30)$. This remarkable difference demonstrates the key relationship between the labellum and cross-pollination. The labellum of $B$. orientale secretes substances to attract and reward the pollinating insects. It is the major and indispensable feature for pollination.

\begin{tabular}{|c|c|c|c|c|c|c|c|c|c|c|c|c|c|c|c|c|c|c|c|c|c|c|c|c|c|}
\hline \multirow{3}{*}{$\begin{array}{c}\text { Plant } \\
\text { no. }\end{array}$} & \multirow{3}{*}{ Treatments } & \multicolumn{24}{|c|}{ Days } \\
\hline & & \multicolumn{3}{|c|}{1} & \multicolumn{3}{|c|}{2} & \multicolumn{3}{|c|}{3} & \multicolumn{3}{|c|}{4} & \multicolumn{3}{|c|}{5} & \multicolumn{3}{|c|}{6} & \multicolumn{3}{|c|}{7} & \multicolumn{3}{|c|}{8} \\
\hline & & $\mathrm{NF}$ & $\mathrm{NFr}$ & RS & $\mathrm{NF}$ & $\mathrm{NFr}$ & RS & $\mathrm{NF}$ & $\mathrm{NFr}$ & RS & $\mathrm{F}$ & $\mathrm{NFr}$ & SS & $\mathrm{F}$ & $\mathrm{NFr}$ & RS & IF & $\mathrm{NFr}$ & RS & IF & $\mathrm{NFr}$ & S & NF & $\mathrm{NFr}$ & RS \\
\hline \multirow{2}{*}{1} & Selfing & 20 & 18 & 90 & 20 & 20 & 100 & 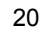 & 18 & 90 & 20 & 18 & 90 & 0 & 20 & 100 & 20 & 19 & 95 & 0 & 20 & 100 & 20 & 20 & 100 \\
\hline & Cross & 20 & 19 & 95 & 20 & 19 & 95 & 20 & 18 & 90 & 20 & 18 & 90 & 20 & 20 & 100 & 20 & 19 & 95 & 20 & 20 & 100 & 20 & 20 & 100 \\
\hline \multirow{2}{*}{2} & Selfin & 20 & 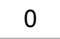 & 0 & 20 & 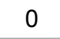 & 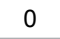 & 20 & 0 & 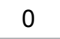 & 20 & 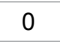 & 8 & 20 & 0 & 0 & 20 & 0 & 0 & 20 & 0 & 0 & 20 & 0 & 0 \\
\hline & oss & 20 & 17 & 85 & 20 & 19 & 95 & 20 & 17 & 85 & 20 & 18 & 90 & 20 & 20 & 100 & 20 & 20 & 100 & 20 & 20 & 100 & 20 & 20 & 100 \\
\hline \multirow{2}{*}{3} & Selfing & 20 & 19 & 95 & 20 & 18 & 90 & 20 & 18 & 90 & 20 & 17 & 85 & 20 & 17 & 85 & 20 & 17 & 85 & 20 & 19 & 95 & 20 & 19 & 95 \\
\hline & Cross & 20 & 20 & 100 & 20 & 19 & 95 & 20 & 19 & 95 & 20 & 17 & 85 & 20 & 17 & 85 & 20 & 7 & 85 & 20 & 19 & 95 & 20 & 19 & 95 \\
\hline \multirow{2}{*}{4} & Selfing & 20 & 16 & 80 & 20 & 17 & 85 & 20 & 19 & 95 & 20 & 17 & 85 & 20 & 19 & 95 & 20 & 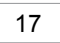 & 85 & 20 & 18 & 0 & 0 & 17 & 35 \\
\hline & Cross & 20 & 17 & 85 & 20 & 19 & 95 & 20 & 20 & 100 & 20 & 18 & 90 & 20 & 19 & 95 & 20 & 17 & 85 & 20 & 18 & 90 & 20 & 17 & 35 \\
\hline \multirow{2}{*}{5} & Selfing & 20 & 20 & 100 & 20 & 19 & 95 & 20 & 19 & 95 & 20 & 20 & 100 & 20 & 20 & 100 & 20 & 20 & 100 & 20 & 20 & 100 & 20 & 17 & 85 \\
\hline & Cross & 2 & 20 & 100 & 20 & 18 & 90 & 2 & 18 & 90 & 2 & 20 & 100 & 20 & 20 & 100 & 20 & 20 & 100 & 20 & 20 & 100 & 20 & 17 & 85 \\
\hline \multirow{2}{*}{ 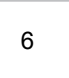 } & Selfing & 20 & 0 & 0 & 20 & 0 & 0 & 20 & 0 & 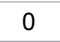 & 20 & 0 & 0 & 20 & 0 & O & 20 & 0 & 0 & 20 & 0 & 0 & 20 & 0 & 0 \\
\hline & Cross & 20 & 19 & 95 & 20 & 19 & 95 & 20 & 17 & 85 & 20 & 19 & 95 & 20 & 17 & 85 & 20 & 20 & 100 & 20 & 20 & 100 & 20 & 20 & 100 \\
\hline & Selfing & 20 & 18 & 90 & 20 & 18 & 90 & 20 & 18 & 90 & 20 & 19 & 95 & 20 & 1 & 85 & 20 & 9 & 95 & 20 & 9 & 95 & 20 & 19 & 95 \\
\hline & Cross & 20 & 17 & 85 & 20 & 18 & 90 & 20 & 18 & 90 & 20 & 18 & 90 & 20 & 17 & 85 & 20 & 19 & 95 & 20 & 19 & 95 & 20 & 19 & 95 \\
\hline & Selfing & 20 & 19 & 95 & 20 & 17 & 85 & 20 & 18 & 90 & 20 & 17 & 85 & 20 & 19 & 95 & 20 & 20 & 100 & 20 & 8 & 90 & 20 & 18 & 90 \\
\hline & Cross & 20 & 18 & 90 & 20 & 18 & 90 & 20 & 19 & 95 & 20 & 18 & 90 & 20 & 19 & 95 & 20 & 20 & 100 & 20 & 18 & 90 & 20 & 18 & 90 \\
\hline & Selfing & 20 & 18 & 90 & 20 & 19 & 95 & 20 & 20 & 100 & 20 & 19 & 95 & 20 & 20 & 100 & 20 & 19 & 95 & 20 & 17 & 85 & 20 & 17 & 85 \\
\hline & oss & 20 & 19 & 95 & 20 & 19 & 95 & 20 & 20 & 100 & 20 & 19 & 95 & 20 & 20 & 100 & 20 & 19 & 95 & 20 & 17 & 85 & 20 & 17 & 85 \\
\hline \multirow{2}{*}{10} & Selfing & 20 & 17 & 85 & 20 & 20 & 100 & 20 & 19 & 95 & 20 & 20 & 100 & 20 & 19 & 95 & 20 & 18 & 90 & 20 & 19 & 95 & 20 & 20 & 100 \\
\hline & Cross & 20 & 20 & 100 & 20 & 20 & 100 & 20 & 19 & 95 & 20 & 20 & 100 & 20 & 19 & 95 & 20 & 18 & 90 & 20 & 19 & 95 & 20 & 20 & 100 \\
\hline
\end{tabular}

$\mathrm{NF}=$ number of flowers; $\mathrm{NFr}=$ number of fruits; $\mathrm{RS}=$ rate of fruit set $(\%)$.

Table 1: Fruit-set rates of different plants pollinated at different times. 


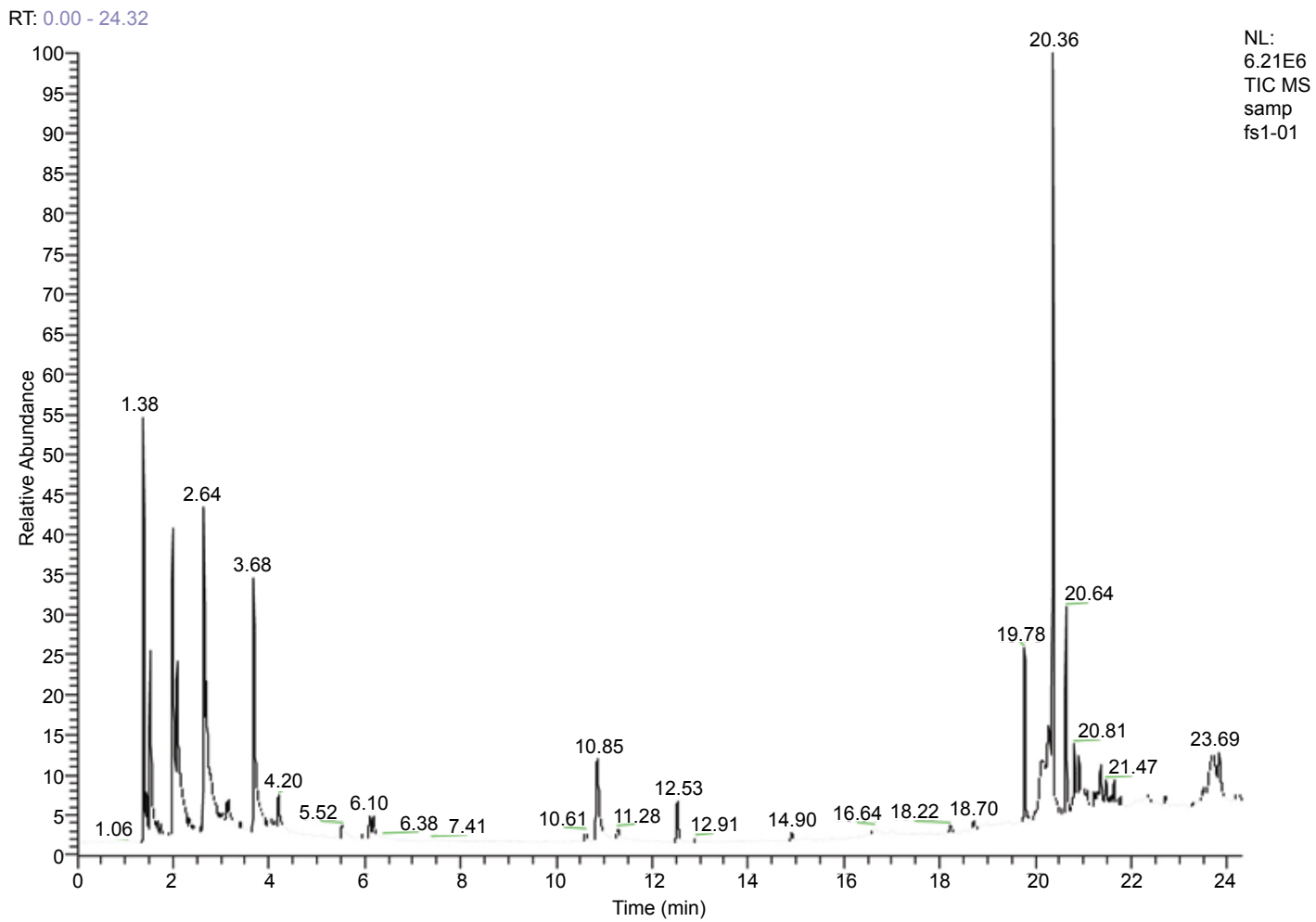

Figure 2: Gas chromatogram of the floral odor of Bulbophyllum orientale..

\begin{tabular}{|c|c|c|c|c|}
\hline \multirow{2}{*}{ Plant type } & \multirow{2}{*}{ Treatments } & \multirow{2}{*}{ Number of flowers } & \multicolumn{2}{|c|}{ Fruit sets } \\
\hline & & & Mean $(\%)$ & SD $(\%)$ \\
\hline \multirow{5}{*}{ Self-compatibility } & Open pollination (natural) & 450 & 10.89 & 6.43 \\
\hline & Bagged flowers & 450 & 0 & 0 \\
\hline & Experimentally outcrossed (bagged) & 450 & 91.55 & 6.30 \\
\hline & Experimentally selfed (bagged) & 450 & 90.43 & 6.93 \\
\hline & Anthers removed in bud (bagged) & 450 & 0 & 0 \\
\hline \multirow{5}{*}{ Self-incompatibility } & Open pollination (natural) & 450 & 5.78 & 6.49 \\
\hline & Bagged flowers & 450 & 0 & 0 \\
\hline & Experimentally outcrossed (bagged) & 450 & 92.22 & 7.44 \\
\hline & Experimentally selfed (bagged) & 450 & 0 & 0 \\
\hline & Anthers removed in bud (bagged) & 450 & 0 & 0 \\
\hline
\end{tabular}

Table 2: Comparison of fruit-set rates under different experimental pollination treatments (mean \pm s.d.).

\section{Breeding system}

No difference was observed between the fruit-set rate for artificial self-pollination $(90.43 \% \pm 6.93 \%, n=30)$ and that for artificial crosspollination $(91.55 \% \pm 6.30 \%, n=30)$ for self-compatible plants $(t=0.65631, d . f .=58, P>0.05)$. The absence of differences indicates that both selfing and crossing are effective breeding methods for $B$. orientale. The fruit-set rate of the flowers that were bagged before opening was zero. This result indicates that $B$. orientale cannot achieve sexual reproduction by automatic self-pollination or generate asexual seeds by apomixis under natural conditions. The stamenless flowers bagged before opening had no seed set, which also confirmed the absence of apomixis. The difference between the control and the artificial selfpollination and more notably that between the control $(10.89 \% \pm 6.43 \%$, $n=30)$ and the artificial cross-pollination $(t=49.1082$, d. $f .=58, P<0.01)$ revealed the shortage of pollinators for complete insemination under natural conditions. Therefore, the mating system of the self-compatible B. orientale plants is a hybrid of crossing and selfing.

For the self-incompatible plants, both the bagged natural flowers and the bagged stamenless flowers had no seed set. No difference exists between the cross-pollination of the self-incompatible plants $(92.22 \% \pm 7.44 \%, n=30)$ and that of the self-compatible plants $(t=0.37453, d . f .=58, P>0.05)$. The fruit-set rates of self-pollination and same-plant cross-pollination were zero. This finding indicates that these plants are self-incompatible and that the mating system is strict crossing. The natural fruit-set rate of the self-incompatible plants $(5.78 \% \pm 6.49 \%, n=30)$ was substantially different from that of the self-compatible plants $(t=3.06498, d . f .=58, P<0.01)$. This result confirms that the insects effect the same-plant cross-pollination and that the fruit-set rate differs because of self-incompatibility and selfcompatibility Table 2 . 


\section{Inbreeding depression}

The seed number and seed germination rate of each fruit from selfing and crossing were calculated Table 3.

No difference was found between selfing and crossing either in the seed number $(\mu=0.1156, d . f .=78, P>0.05)$ or in the seed germination rate $(\mu=0.09888, d . f .=78, P>0.05)$. Compared with self-incompatible plants, the self-compatible plants could use insect pollination much more effectively to produce more seeds. This result suggests that selfing can increase the seed number of the population. Using the seed amount to calculate selfing relative fitness, $\mathrm{Ws} / \mathrm{Wc}=0.9763$, inbreeding depression $\delta=0.024$.

\section{Pollinator shortage rate and selfing rate}

Table 4 shows the pollinator shortage rate and the selfing rate of each population. The natural fruit-set rate of $B$. orientale was $10.89 \%$, and the crossing and selfing rates were $5.50 \%$ (50.51\% of the total fruitset rate) and $5.39 \%$ (49.49\% of the total fruit-set rate), respectively. The pollinator shortage rate $=1-10.89 / 91.55=88.10 \%$. Thus, the behavior of the pollinating insects induced the same-plant cross-pollination in $B$. orientale. The percentages of self-pollination and cross-pollination were almost equal. The cross-pollinator (behavior) shortage rate of the inflorescences $=1-5.50 / 91.55=93.99 \%$. The pollinator shortage rate within the population $=1-8.59 / 91.55=90.62 \%$. The inbreeding (within population) rate was $5.11 \%$, the outbreeding (among populations) rate was only $0.34 \%$, and the cross-pollinator (behavior) shortage rate was $99.63 \%(1-0.34 \%)$. These results show that inbreeding (within population) is predominant in B. orientale.

\section{Discussion}

The floral shape is one of the most important aspects of the interaction between plants and pollinators, determining the efficiency of nectar fetching by the pollinator, the pollen attachment, and the pollen acquisition by the stigma from pollinating agents [36]. The observation of flowering phenology and flowering biology of characteristics of $B$. Orientale showed that the structures of the flower, such as the lip and stigma morphology and the anther and stigma position are suitable for pollination by small insects. The longer opening period of the flowers and the pollen viability and stigma receptivity of the overall florescence improve the pollination success rate under the condition of insufficient pollinators. The floral odor they emit, such as 2-copaene and lipoids, emits food signals to the pollinators and attracts their visits. The successful pollination of $B$. orientale depends on the flower's excretion. This phenomenon is similar to putrid berries and thus attracts and rewards pollinating insects to complete pollination on the unique flower structure. The cross-pollination mechanism that utilizes the floral parts and glutinous substance as pollination implements is achieved by a series of ingenious "designs" rather than by the functions of a single component. The unique floral structure has a clear predisposition for cross-pollination. The natural fruit ratio and rate of pollinator scarcity show that both selfing and outcrossing mainly occur within the same population (the fruit ratio is $5.11 \%$ ), while the outbreeding rate of two populations $30 \mathrm{~m}$ apart is $0.34 \%$, which is $6.65 \%$ of the fruit rate within population. Therefore, pollination in natural conditions occurs mainly within a population, and chances of selfing (including geitonogamy) and outbreeding (dioecious) are equal (5.5\% vs. 5.39\%), showing that inbreeding is predominant in $B$. orientale. However, the visiting insects may bring the pollinaria into the stigma cavity of the same inflorescence or a different inflorescence of the same plant because each inflorescence has many flowers. Self-compatible plants using both crossing and selfing could acquire more opportunity in pollination to improve the gross seed output and increase the reproductive assurance coefficient despite the pollinator deficiency. The self-incompatible plants in the populations use strict crossing to prevent self-pollination or same plant cross-insemination. This mixed mating system may have developed to reduce the selective pressure caused by frequent selfing and ensure both the reproduction and the crossing predominance in the populations, forming the cross-mixed mating system. Inbreeding depression may greatly offset the benefits of inbreeding, whereas outbreeding is easily affected by environmental conditions and thus costly (in this experiment, $99.63 \%$ of the flowers were not fertilized). The evolutionary compromise gave rise to the mixed mating system as a trade-off between inbreeding and outbreeding. The self-compatibility and self-incompatibility in the plants and the partial application of same-plant cross-insemination also represent this trade-off. The selfing and crossing offspring of the plants in each population were analyzed by comparing the seed number and the seed germination rate of each capsule to estimate the degree of inbreeding depression. No difference was found, and selfing did not show a higher mating cost than crossing. Therefore, considering seed generation and seed germination rate as

\begin{tabular}{|c|c|c|c|c|}
\hline \multirow{2}{*}{ Years } & \multicolumn{2}{|c|}{ Selfing } & \multicolumn{2}{c|}{ Crossing } \\
\cline { 2 - 5 } & Seed number & Germination rates $(\%)$ & Seed number & Germination rates $(\%)$ \\
\hline 2004 & $45396.25 \pm 10317.87(n=8)$ & $0.39 \pm 0.28(n=8)$ & $29797.50 \pm 10884.57(n=8)$ & $0.39 \pm 0.29(n=8)$ \\
\hline 2005 & $27023.75 \pm 15079.29(n=8)$ & $1.11 \pm 1.31(n=8)$ & $32245.00 \pm 10497.98(n=8)$ & $1.05 \pm 1.26(n=8)$ \\
\hline 2006 & $24792.38 \pm 4348.35(n=8)$ & $0.39 \pm 0.27(n=8)$ & $48751.25 \pm 11445.22(n=8)$ & $0.41 \pm 0.28(n=8)$ \\
\hline 2007 & $45632.13 \pm 10927.42(n=8)$ & $0.95 \pm 1.38(n=8)$ & $34046.25 \pm 13473.94(n=8)$ & $0.91 \pm 1.32(n=8)$ \\
\hline 2008 & $45146.63 \pm 6146.48(n=8)$ & $0.40 \pm 0.26(n=8)$ & $44860.25 \pm 7441.85(n=8)$ & $0.37 \pm 0.26(n=8)$ \\
\hline $\bar{x} \pm$ SD & $37598.23 \pm 13620.02(n=40)$ & $0.65 \pm 0.89(n=40)$ & $37940.05 \pm 12815.81(n=40)$ & $0.63 \pm 0.85(n=40)$ \\
\hline
\end{tabular}

Table 3: Seed number and seed germination rate for selfing and crossing.

\begin{tabular}{|c|c|c|c|c|c|c|c|c|c|c|}
\hline \multirow{2}{*}{ Year } & \multirow{2}{*}{$\mathrm{AO}$} & \multicolumn{3}{|c|}{ Within inflorescence } & \multicolumn{3}{|c|}{ Within population } & \multicolumn{3}{|c|}{ Among populations } \\
\hline & & IN & AR & Sh & IN & AR & $\mathrm{Se}$ & IN & AR & Sh \\
\hline 2006 & 92.66 & 10.67 & 5.39 & 88.48 & 8.93 & 3.09 & 5.84 & 9.00 & 0.41 & 90.29 \\
\hline 2007 & 90.00 & 11.33 & 5.52 & 87.41 & 8.51 & 3.32 & 5.19 & 8.13 & 0.32 & 90.97 \\
\hline 2008 & 92.00 & 10.67 & 5.60 & 88.40 & 8.32 & 4.01 & 4.31 & 8.02 & 0.28 & 91.28 \\
\hline $\bar{x}$ & 91.55 & 10.89 & 5.50 & 88.10 & 8.59 & 3.47 & 5.11 & 8.38 & 0.34 & 90.85 \\
\hline
\end{tabular}

$\mathrm{AO}=$ artificial outcrossing; $\mathrm{IN}=$ in natural; $\mathrm{AR}=$ anthers removed in bud; Sh = shortage rate (\%); Se = selfing rate (\%).

Table 4: Pollinator shortage rate and selfing rate of Bulbophyllum orientale. 


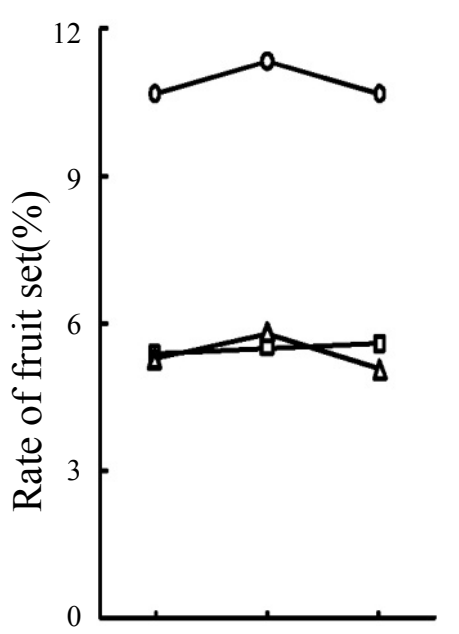

'06

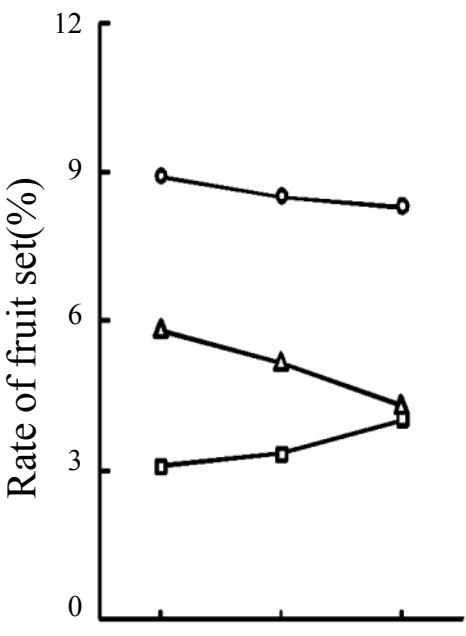

'06
‘07

B

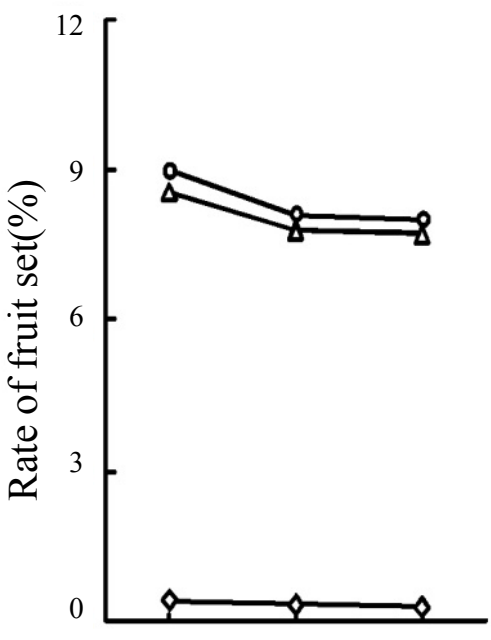

'06 '07 '08

C

Figure 3: Natural $(-\circ-)$, crossing $(-\Delta)$, selfing $(-\square)$, and among populations $(\neg-)$ fruit-set rates. (A) Natural selfing fruit-set rate in inflorescences with anthers removed in bud. (B) Natural selfing fruit-set rate in populations with anthers removed in bud. (C) Natural selfing fruit-set rate among populations with anthers removed in bud.

indicators of inbreeding depression, selfing would evolve as long as pollinators are lacking. This phenomenon is attributed to the absence of high costs in pollen and seed discounting Figure 2.

The change in pollination environment in the three flowering seasons was recorded. The fruit-set rates of natural, stamenless, and cross-unabridged flowers were compared Figure 3. A significant difference was found between the cross-pollination rate and the selfpollination rate within and among the flowering seasons. The low visiting rate of cross-pollinators led to the remarkably low fruit-set rate of stamenless flowers and self-incompatible flowers. More importantly, the difference in the fruit-set rate among the stamenless flowers, selfincompatible flowers, and unabridged flowers demonstrated that selfing of different flowers on the same plant could increase the fruit-set rate when pollinators were lacking or absent. The absence of pollination in many flowers during the flowering season suggested that cross- and self-pollination were both extremely rare. The breeding assurance that persisted in the biological environment of the $B$. orientale population resulted in the evolution of $B$. orientale from strict crossing to mixed mating. Therefore, the existence of $3.5 \%$ of individuals in the population as self-incompatible can be considered a remnant of strict crossing, showing that $B$. orientale originally used a strategy of strict crossing. Given the low inbreeding depression $(\delta=0.024)$, strict crossing is broken down by the lack of pollinators. However, retaining some strict crossing individuals would benefit by balancing or by preventing over-selfing. We compared the non-seed-set percentage of the stamenless flowers (or self-incompatible flowers) under natural conditions with that of the artificially cross-pollinated flowers to quantify the visiting rate of pollinators. During the 3 year observation, only some of the flowers in the populations had seed set after being visited, regardless of how the pollinators changed. The selfing rate fluctuated only in a small range among populations and with time Figure 3. Consistently, almost $90 \%$ of the flowers in the populations were not pollinated, regardless of how many flowers were visited by pollinators each year, and the crossing rate was always low. The pollination rate (including the self-pollination rate) still has a rather large potential for improvement, and the concurrence of selfing and crossing is ecologically favorable.
The data thoroughly demonstrated the occurrence and maintenance of the cross-mixed mating system affected by pollinating behavior. The pollinators successively visited neighboring flowers and inflorescences of self-compatible and self-incompatible plant populations. These flowers and inflorescences had many clones, and each clone had many multi-flower inflorescences. The findings not only illustrate the reproductive countermeasure of selfing in the mating system evolution of $B$. orientale but also extended the significance of our research to the evolution of the mixed mating system. In wild populations, uncertainty in the pollinator environment is normal $[9,27,37]$. The developmental mechanism of the flower not only promotes crossing but also maximizes the pollinator-mediated crossing and affects partial selfing. This phenomenon addresses the uncertain pollinator environment and maintains both the advantage of crossing and the breeding assurance of selfing [9]. Thus, a cross-mixed mating system that fluctuates with a varying pollinator environment is developed and maintained.

\section{Acknowledgments}

This project was financially supported by the National Program for Wild Life Conservation and Nature Reserve Construction of China and by the Science and Technology Plan of Shenzhen (Grant no. 2006-464). We thank Dr. Daojian Yu of the Shenzhen Inspection and Quarantine Bureau for the identification of insect specimens. We also thank Xu-Hui Chen and Wei-Rong Liu for assistance in the field observations.

\section{References}

1. Les DH (1988) Breeding systems, population structure and evolution in hygrophilous angiosperms. Annals of the Missouri Botanical Garden 75: 819835

2. Richards AJ (1997) Plant breeding systems, (Edn 2) Chapman \& Hall, London, UK

3. Li QJ, Xu ZF, Kress WJ, Xia YM, Zhang L, et al. (2001) Pollination: flexible style that encourages outcrossing. Nature 410: 432.

4. Li QJ, Kress WJ, Xu ZF, Xia YM, Zhang L, et al. (2002) Mating system and stigmatic behavior during flowering of Alpinia Kwangsiensis (Zingiberaceae) Plant Systematics and Evolution 232: 123-132.

5. Stebbins GL (1957) Self-fertilization and population variability in the higher plants. American Naturalist 91: 337-354. 
Citation: Chen LJ, Zhang GQ, Li LQ, Zhang YT, Rao WH (2014) Development and Maintenance of a Cross-mixed Mating System in the Orchid Bulbophyllum orientale. J Phylogen Evolution Biol 2: 124. doi:10.4172/2329-9002.1000124

Page 8 of 8

6. Liu ZJ, Chen LJ, Liu KW, Li LQ, Ma XY, et al. (2008b) Chenorchis, a new orchid genus and its eco-strategy of ant pollination. Acta Ecologica Sinica 28: 24332444

7. Darwin C (1876) The effect of cross and self fertilization in the Vegetable Kingdom. Murray, London.

8. Piper JG, Charlesworth B, Charlesworth D (1984) A high rate of self-fertilization and increased seed fertility of homostyle primroses. Nature 310: 50-51.

9. Liu KW, Liu ZJ, Huang L, Li LQ, Chen LJ, et al. (2006) Pollination: selffertilization strategy in an orchid. Nature 441: 945-946.

10. Chen LJ, Liu KW, Xiao XJ, Tsai WC, Hsiao YY, et al. (2012) The anther steps onto the stigma for self-fertilization in a slipper orchid. PLoS One 7: e37478.

11. Jain SK (1976) The evolution of inbreeding in plants. Annual Review of Ecology and Systematics 7: 469-495.

12. Stebbins GL (1970) Adaptive radiation of Reproductive Characteristics in angiosperms. I. Pollination mechanisms. Annual Review of Ecology and Systematics 1: 307-326.

13. Liu KW, Liu ZJ, Lei SP, Li LQ, Chen LJ, et al. (2005) Study on pollination biology in Paphiopedilum armeniacum (Orchidaceae). Shenzhen Science \& Technology 139: 171-183.

14. Finer MS, Morgan MT (2000) Geitonogamy and the evolution of inflorescence design in Asclepias speciosa. American Journal of Botany 87: 53.

15. Liu ZJ, Chen LJ, Rao WH, Li LQ, Zhang YT (2008a) Correlation between numeric dynamics and reproductive behavior in Cypripedium lentiginosum. Acta Ecologica Sinica 28: 111-121.

16. Yao H, Luo YB (2011) Pollen limitation and variation in floral longevity in gynodioecious Potentilla tanacetifolia. Journal of Systematics and Evolution 49: 539-545.

17. He YP, Liu JQ (2003) A review on recent advances in the studies of plan breeding system. Acta Phytoecologica Sinica 27: 151-163.

18. Fan JH, Thien LB, Luo YB (2011) Pollination systems, biogeography, and divergence times of three allopatric species of Schisandra in North America, China, and Japan. Journal of Systematics and Evolution 49: 330-338.

19. Holsinger KE (1991) Inbreeding depression and the evolution of plant mating systems. Trends Ecol Evol 6: 307-308.

20. Chang SM, Rausher MD (1998) Frequency-dependent pollen discounting contributes to maintenance of a mixed mating system in the common morning glory Ipomoea purpurea. Am Nat 152: 671-683.

21. Barrett SC, Harder LD (1996) Ecology and evolution of plant mating. Trends Ecol Evol 11: 73-79.
22. Zheng GL, Li P, Pemberton RW, Luo YB (2011) Mixed bumblebee and blowfly pollination of Cypripedium flavum (Orchidaceae) in Sichuan, China. Ecological Research 26: 453-459

23. Liu ZJ, Chen LJ, Liu KW, Li LQ, Rao WH, et al. (2013) Adding perches for cross-pollination ensures the reproduction of a self-incompatible orchid. PLoS One 8: e53695.

24. Darwin C (1890) The various contrivances by which orchids are fertilized by insects. (Edn 2). Murray, London.

25. Li P, Pemberton R, Zhen G, Luo YB (2012) Fly pollination in Cypripedium: a case study of sympatric $C$. sichuanensis and $C$. microatnhum. Botanical Journal of the Linnean Society 170: 50-58.

26. Liu ZJ, Chen LJ, Liu KW, Li LQ, Rao WH (2010) A floral organ moving like a caterpillar for pollinating. Journal of Systematics and Evolution 48: 102-108.

27. Liu ZJ, Chen LJ, Lei SP, Rao WH, Li LQ (2007) Reproduction strategy of Trias verrucosa (Orchidaceae) from China. Acta Ecologica Sinica 27: 4460-4468.

28. Kalisz S, Vogler DW, Hanley KM (2004) Context-dependent autonomous selffertilization yields reproductive assurance and mixed mating. Nature 430: 884 887

29. Tsi ZH (1999) Flora Reipublicae Popularis Sinicae 19. Beijing: Science Press.

30. Dafni A (1992) Pollination ecology: A practical approach. New York: Oxford University Press.

31. Rodriguez-Riaño T, Dafni A (2000) A new procedure to assess pollen viability. Sexual Plant Reproduction 12: 241-244

32. Lande R, Schemske DW (1985) The evolution of self-fertilization and inbreeding depression in plants. I. Genetic models. Evolution 39: 24-40.

33. Charlesworth D, Morgan MT, Charlesworth B (1990) Inbreeding depression, genetic load, and the evolution of outcrossing rates in a multilocus system with no linkage. Evolution 44: 1469-1489.

34. Smithson A (2006) Pollinator limitation and inbreeding depression in orchid species with and without nectar rewards. New Phytol 169: 419-430.

35. Qin JD (1987) The Relationships between Insects and Plants. Beijing: Science press.

36. Sakai S, Kato M, Inoue T (1999) Three pollination guilds and variation in floral characteristics of Bornean gingers (Zingiberaceae and Costaceae). Am J Bot 86: $646-658$

37. Burd M (1994) Bateman's principle and plant reproduction: the role of pollen limitation in fruit and seed set. Botanical Review 60: 83-139. 